\title{
Image De-noising Using Multilevel Filtering Approach
}

\author{
Shiva Shrivastava ${ }^{\#}$, Rahul Dubey ${ }^{\#}$, S.G.Kerhalker ${ }^{\#}$ \\ \# Oriental Institute of Science and Technology, Bhopal
}

\begin{abstract}
Visual information transmitted in the form of digital images is becoming a major method of communication in the modern age, but the image obtained after transmission is often corrupted with noise. The received image needs processing before it can be used in applications. Image denoising involves the manipulation of the image data to produce a visually high quality image. Salt \& pepper (Impulse) noise and the additive white Gaussian noise and blurredness are the types of noise that occur during transmission and capturing. To remove these types of noise we have many filters like mean filter, median filter, inverse filter, wiener filter. No single one filter can remove both types of noise. A new approach for de-noising based on multilevel filter is proposed in this paper. The numerical computation has been done using MATLAB 7.8.0.

Keywords: Peak Signal to noise ratio, Image denoising, Image restoration, Filter.
\end{abstract}

\section{Introduction}

Image denoising is an important image processing task, both as a process itself, and as a component in other processes. Very many ways to denoise an image or a set of data exists. The main properties of a good image denoising model are that it will remove noise while preserving edges.

Image denoising is often used in the field of photography or publishing where an image was somehow degraded but needs to be improved before it can be printed. For this type of application we need to know something about the degradation process in order to develop a model for it. When we have a model for the degradation process, the inverse process can be applied to the image to restore it back to the original form.

An image is often corrupted by noise in its acquition and transmission. Image denoising is used to remove the additive noise while retaining as much as possible the important signal features. In the recent years there has been a fair amount of research on wavelet thresholding and threshold selection for signal de-noising [1], [2], because wavelet provides an appropriate basis for separating noisy signal from the image signal. The motivation is that as the wavelet transform is good at energy compaction, the small coefficient are more likely due to noise and large coefficient due to important signal features. These small coefficients can be thresholded without affecting the significant features of the image.

Thresholding is a simple non- linear technique, which operates on one wavelet coefficient at a time. In its most basic form, each coefficient is thresholded by comparing against threshold, if the coefficient is smaller than threshold, set to zero; otherwise it is kept or modified. Replacing the small noisy coefficients by zero and inverse wavelet transform on the result may lead to reconstruction with the essential signal characteristics and with less noise.

Since the work of Donoho \& Johnstone [1], [3], [4], there has been much research on finding thresholds, however few are specifically designed for images. In this paper, a near optimal threshold estimation technique for image denoising is proposed which is subband dependent i.e. the parameters for computing the threshold are estimated from the observed data, one set for each subband.

\section{Literature Survey}

A novel switching median filter incorporating with a powerful impulse noise detection method, called the boundary discriminative noise detection (BDND), was proposed by Pei-Eng Ng; Kai-Kuang Ma for effectively denoising extremely corrupted images. To determine whether the current pixel is corrupted, the proposed BDND algorithm [5] first classifies the pixels of a localized window, centering on the current pixel, into three groups-lower intensity impulse noise, uncorrupted pixels, and higher intensity impulse noise. The center pixel will then be considered as "uncorrupted," provided that it belongs to the "uncorrupted" pixel group, or "corrupted." For that, two boundaries that discriminate these three groups require to be accurately determined for yielding a very high noise detection accuracy-in our case, achieving zero miss -detection rate while maintaining a fairly low false-alarm rate, even up to $70 \%$ noise corruption. Four noise models are considered for performance evaluation. Extensive simulation results conducted on both monochrome and color images under a wide range (from $10 \%$ to $90 \%$ ) of noise corruption clearly show that our proposed switching median filter substantially outperforms all existing median-based filters, in terms of suppressing impulse noise while preserving image details, and yet, the proposed BDND is algorithmically simple, suitable for real-time implementation and application (2009). 
A new switch median filter is presented by B.GHANDEHARIAN, and colleagues for suppression of impulsive noise in image. The proposed filter is Modified Adaptive Center Weighted Median (MACWM) filter [6] with an adjustable central weight obtained by partitioning the observation vector space. Dominant points of the proposed approach are partitioning of observation vector space using clustering method, training procedure using LMS algorithm then freezing weights in each block are applied to test image.

A new approach for restoring impulse noise corrupted images by Zhu Zhu, Qing Wang, Xiaoguo Zhang (2011) [7]. It uses the standard deviation to find out the proximate 5 pixels in the filter window. Subsequently, the weighted mean value and the weighted standard deviation of the 5 pixels are used to obtain thresholds. Using the thresholds we identify the noise pixels, and then restore them with the median of the 5 pixels and the weighed mean of them.

Lakhwinder Kaur, Savita Gupta, R.C. Chauhan, in 2008 proposes an adaptive threshold estimation method for image denoising in the wavelet domain based on the generalized Guassian distribution (GGD) modeling of subband coefficients. The proposed method[8] called NormalShrink iscomputationally more efficient and adaptive because the parameters required for estimating the threshold depend on subband data.

Zhang. L, et.al an efficient image denoising scheme by using principal component analysis (PCA) with local pixel grouping (LPG). For a better preservation of image local structures, a pixel and its nearest neighbors are modeled as a vector variable, whose training samples are selected from the local window by using block matching based LPG. Such an LPG procedure guarantees that only the sample blocks with similar contents are used in the local statistics calculation for PCA transform estimation, so that the image local features can be well preserved after coefficient shrinkage in the PCA domain to remove the noise [9] .

\section{Types ff of Noises}

Any real world sensor is a ected by a certain degree of noise, whether it is thermal, electrical or otherwise. This noise will corrupt the true measurement of the signal, such that any resulting data is a combination of signal and noise.

Additive noise, probably the most common type, can be expressed as:

$$
I(t)=S(t)+N(t)
$$

Where $\mathrm{I}(\mathrm{t})$ is the resulting data measured at time $\mathrm{t}, \mathrm{S}(\mathrm{t})$ is the original signal measured, and $\mathrm{N}(\mathrm{t})$ is the noise introduced by the sampling process, environment and other sources of interference.

defined by the mean ${ }^{-} \mathrm{x}$ and standard deviationo. Example. At each input voxel vin, a sample is taken from the normal variate distribution $\mathrm{G}(\mathrm{d})$, and added to the image to produce. For some mean noise ${ }^{-} \mathrm{x}$ and standard deviation $\sigma$. The seed $d$ is an arbitrary number to start the pseudo-random sequence. For applications that require high entropy, a common technique is to seed with the current system time (in milliseconds)

\section{Salt and Pepper Noise}

Salt and pepper noise is an impulse type of noise, which is also referred to as intensity spikes. This is caused generally due to errors in data transmission. It has only two possible values, $a$ and $b$. The probability of each is typically less than

0.1. The corrupted pixels are set alternatively to the minimum or to the maximum value, giving the image a "salt and pepper" like appearance. Unaffected pixels remain unchanged. For an 8-bit image, the typical value for pepper noise is 0 and for salt noise 255 . .

The salt and pepper noise is generally caused by malfunctioning of pixel elements in the camera sensors, faulty memory locations, or timing errors in the digitization process.

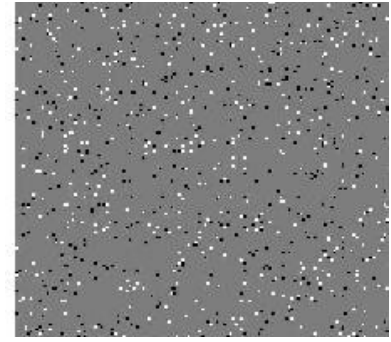

Fig 1. Image affected by Salt $\mathrm{n}$ pepper noise 


\section{Types of Filters}

Basically there are two types of filter generally used in image de-noising:

1. Linear Filter

2. Non-Linear Filter

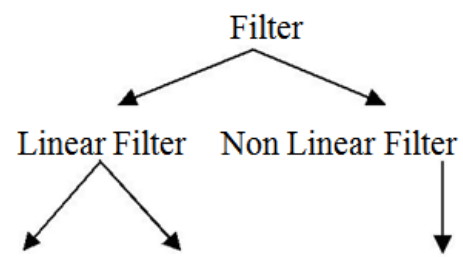

Fig2. Filter classification

Additive Gaussian Noise: In Gaussian noise models, the noise density follows a Gaussian normal distribution $\mathrm{G}(\mathrm{x}, \sigma)$

\section{Linear Filtering Mean Filter}

A mean filter [Um98] acts on an image by smoothing it; that is, it reduces the intensity variation between adjacent pixels. The mean filter is nothing but a simple sliding window spatial filter that replaces the center value in the window with the average of all the neighboring pixel values including itself. By doing this, it replaces pixels that are unrepresentative of their surroundings. It is implemented with a convolution mask, which provides a result that is a weighted sum of the values of a pixel and its neighbors.

It is also called a linear filter. The mask or kernel is a square. Often a $3 \times 3$ square kernel is used. If the coefficients of the mask sum up to one, then the average brightness of the image is not changed. If the coefficients sum to zero, the average brightness is lost, and it returns a dark image. The mean or average filter works on the shift-multiply-sum principle.

Image 3.1 is the one corrupted with salt and pepper noise with a variance of 0.05 .

The output image after Image 3.1 is subjected to mean filtering is shown in Image 3.2.

It can be observed from the output that the noise dominating in Image 3.1 is reduced in Image 3.2. The white and dark pixel values of the noise are changed to be closer to the pixel values of the surrounding ones. Also, the brightness of the input image remains unchanged because of the use of the mask, whose coefficients sum up to the value one.

The mean filter is used in applications where the noise in certain regions of the image needs to be removed. In other words, the mean filter is useful when only a part of the image needs to be processed.

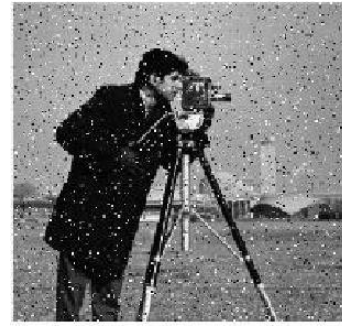

Fig3.1 Image with noise

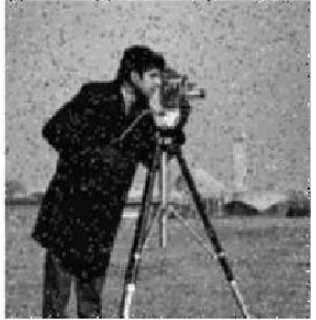

Fig3.2 Mean Filtered Image

Such filters are known for their ability in automatically tracking an unknown circumstance or when a signal is variable with little a priori knowledge about the signal to be processed [Li93]. In general, an adaptive filter iteratively adjusts its parameters during scanning the image to match the image generating mechanism. This mechanism is more significant in practical images, which tend to be non- stationary. Compared to other adaptive filters, the Least Mean Square (LMS) adaptive filter is known for its simplicity in computation and implementation. The basic model is a linear combination of a stationary low-pass image and a non-stationary high-pass component through a weighting function [Li93]. Thus, the function provides a compromise between resolution of genuine features and suppression of noise.

The LMS adaptive filter incorporating a local mean estimator [Li93] works on the following concept. 
$A$ window, $W$, of size $m \times n$ is scanned over the image. The mean of this window, $\mu$, is subtracted from the elements in the window to get the residual matrix $\mathrm{Wr}$

$W r=W-\mu$

The largest eigenvalue $\lambda$ of the original window is calculated from the autocorrelation matrix of the window considered. The use of the largest eigenvalue in computing the modified weight matrix for the next iteration reduces the minimum mean squared error. A value $\eta$ is selected such that it lies in the range $(0,1 / \lambda)$. In other words,

$0<\eta<1 / \lambda$

When the image is corrupted with salt and pepper noise, it looks as shown in Image 4.1. When Image 4.1 is subjected to the LMS adaptive filtering, it gives an output image shown in Image 4.2. Similar to the mean filter, the LMS adaptive filter works well for images corrupted with salt and pepper type noise. But this filter does a better denoising job compared to the mean filter.

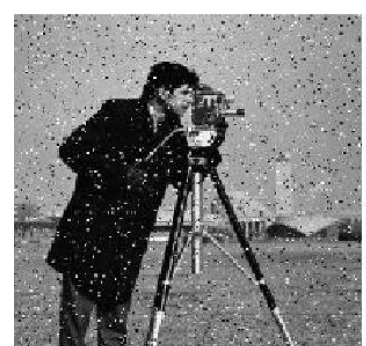

Fig4.1 Image with noise

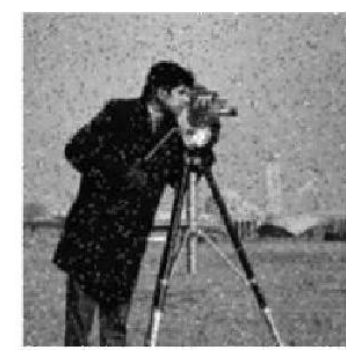

Fig4.2 LMS filtered image

\section{LMS Adaptive Filter}

An adaptive filter does a better job of denoising images compared to the averaging filter. The fundamental difference between the mean filter and the adaptive filter lies in the fact that the weight matrix varies after each iteration in the adaptive filter while it remains constant throughout the iterations in the mean filter.Adaptive filters are capable of denoising non- stationary images, that is, images that have abrupt changes in intensity.

\section{Non-Linear Filtering Median Filter}

A median filter belongs to the class of nonlinear filters unlike the mean filter. The median filter also follows the moving window principle similar to the mean filter. A $3 \times 3,5 \times 5$, or $7 \times 7$ kernel of pixels is scanned over pixel matrix of the entire image. The median of the pixel values in the window is computed, and the center pixel of the window is replaced with the computed median. Median filtering is done by, first sorting all the pixel values from the surrounding neighborhood into numerical order and then replacing the pixel being considered with the middle pixel value. Note that the median value must be written to a separate array or buffer so that the results are not corrupted as the process is performed. Figure 5.1, and Fig 5.2 illustrates the methodology

\section{Proposed Approach}

A new hybrid approach for image de-noising is present in this paper based on the multilevel filtering. This filter is the combination of Pseudo-inverse filter and wiener filter. When we arrange these filters in series we get the desired output. First we remove the white Gaussian noise and then pass the result to the wiener filter.

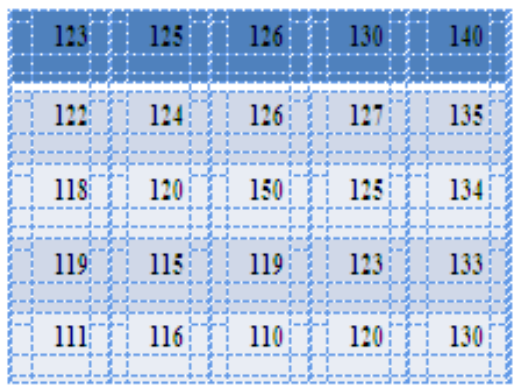

Neighborhood values:

$115,119,120,123,124,125,126,127,150$

Median value: 124 
The median is more robust compared to the mean. Thus, a single very unrepresentative pixel in a neighborhood will not affect the median value significantly. Since the median value must actually be the value of one of the pixels in the neighborhood, the median filter does not create new unrealistic pixel values when the filter straddles an edge. For this reason the median filter is much better at preserving sharp edges than the mean filter. These advantages aid median filters in denoising uniform noise as well from an image.

As mentioned earlier, the image "cameraman.tif" is corrupted with salt and pepper noise with with salt and pepper noise and is given to the median filter filtering. The window specified is of size $3 \times 3$. Image is the output after median filtering. It can be observed that the edges are preserved and the quality of denoising is much better compared to the Images.

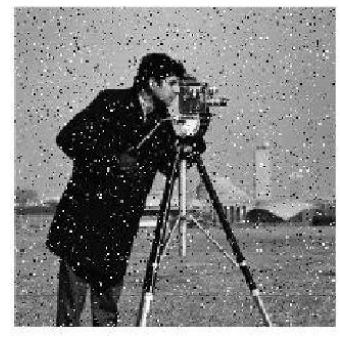

Fig5.1 Image with noise

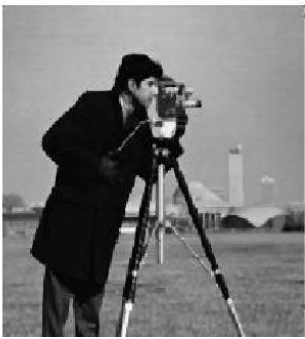

Fig5.2 Median Filtered Image

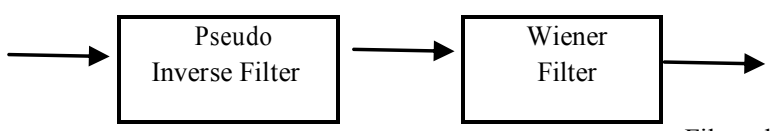

Filtered Image

Noisy Image

Fig5 ImageDe-noising Process

\begin{tabular}{|l|c|c|l|}
\hline Methods & $\begin{array}{c}\text { SNR } \\
\text { Input }\end{array}$ & $\begin{array}{c}\text { SNR } \\
\text { Output }\end{array}$ & \multicolumn{1}{|c|}{ Noise, Var } \\
\hline Mean filter & 18.88 & 27.43 & Salt and pepper, 0.05 \\
\hline Mean filter & 13.39 & 21.24 & Gaussian, 0.05 \\
\hline $\begin{array}{l}\text { LMS } \\
\text { adaptive } \\
\text { filter }\end{array}$ & 18.88 & 28.01 & Salt and pepper, 0.05 \\
\hline $\begin{array}{l}\text { LMS } \\
\text { adaptive } \\
\text { filter }\end{array}$ & 13.39 & $22 . .40$ & Gaussian, 0.05 \\
\hline $\begin{array}{l}\text { Median } \\
\text { filter }\end{array}$ & 18.88 & 47.97 & Salt and pepper, 0.05 \\
\hline $\begin{array}{l}\text { Median } \\
\text { filter }\end{array}$ & 13.39 & 22.79 & Gaussian, 0.05 \\
\hline $\begin{array}{l}\text { Proposed } \\
\text { Filter }\end{array}$ & 18.88 & 44.67 & Salt and pepper, 0.05 \\
\hline $\begin{array}{l}\text { Proposed } \\
\text { Filter }\end{array}$ & 13.39 & 26.28 & Gaussian, 0.05 \\
\hline
\end{tabular}

\section{Conclusion and Future Scope}

We used various images in .jpg format. Adding two noise (Salt-n-pepper noise, Gaussian noise) and apply the noisy image to proposed filter. The final filtered image is depending upon the blurring angle and the blurring length and the percentage of the impulse noise. When these variables are less the filtered image is nearly equal to the original image.

The PSNR is increasing when the noise percentage is less.

\section{References}

[1]. D.L. Donoho, De-Noising by Soft Thresholding, IEEE Trans. Info. Theory 43, pp. 933-936, 1993.

[2]. M. Lang, H. Guo and J.E. Odegard, Noise reduction Using Undecimated Discrete wavelet transform, IEEE Signal Processing Letters, 1995.

[3]. D.L. Donoho and I.M. Johnstone, Adapting to unknown smoothness via wavelet shrinkage, Journal of American Statistical Assoc., Vol. 90, no. 432, pp 1200-1224, Dec. 1995.

[4]. J.L. Starck, E.J. Candes, D.L. Donoho, The curvelet transform for image denoising, IEEE Transaction on Image Processing 11 (6) (2002) 670-684. 
[5]. A switching median filter with boundary discriminative noise detection for extremely corrupted images byPei-Eng Ng; Kai-Kuang Ma.

[6]. Modified Adaptive Center Weighted Median Filter for Suppressing Impulsive Noise in Images by B.GHANDEHARIAN, H.SADOGHI YAZDI and F.HOMAYOUNI, International Journal of Research and Reviews in Applied Sciences (2009)

[7]. A new approach for restoring impulse noise corrupted images by Zhu Zhu, Qing Wang, Xiaoguo Zhang (2011)

[8]. Image Denoising using Wavelet Thresholding by Lakhwinder Kaur Savita Gupta R.C. Chauhan, IEEE (2008)

[9]. Two-stage image denoising by principal component analysis with local pixel grouping by Lei Zhang, WeishengDong, DavidZhang, Guangming Shi.[2010] 